\title{
Secured and progressive transmission of compressed images on the Internet: application to telemedicine
}

\author{
Marie Babel ${ }^{a}$, Benoît Parrein ${ }^{b}$, \\ Olivier Déforges ${ }^{a}$, Nicolas Normand ${ }^{b}$, Jean-Pierre Guédon ${ }^{b}$ and Joseph Ronsin ${ }^{a}$ \\ ${ }^{a}$ IETR UMR CNRS 6164 - Image and Remote Sensing Group / INSA of Rennes, \\ 20 av. des Buttes de Coësmes - CS 14315, 35043 Rennes, France; \\ ${ }^{b}$ IRCCyN CNRS UMR 6795, Image and Videocommunications Team - Mojette group, \\ La Chantrerie, rue Christian Pauc, BP 50609, 44306 Nantes Cédex 3, France
}

\begin{abstract}
Within the framework of telemedicine, the amount of images leads first to use efficient lossless compression methods for the aim of storing information. Furthermore, multiresolution scheme including Region of Interest (ROI) processing is an important feature for a remote access to medical images. What is more, the securization of sensitive data (e.g. metadata from DICOM images) constitutes one more expected functionality: indeed the lost of IP packets could have tragic effects on a given diagnosis. For this purpose, we present in this paper an original scalable image compression technique (LAR method) used in association with a channel coding method based on the Mojette Transform, so that a hierarchical priority encoding system is elaborated. This system provides a solution for secured transmission of medical images through low-bandwidth networks such as the Internet.
\end{abstract}

Keywords: LAR method, medical image compression, Mojette transform, multiresolution, priority encoding system.

\section{INTRODUCTION}

The emergence of new easy, fast and reliable techniques of image storage and Internet transmission have stirred up the practice of medicine. For example, patients could get immediate diagnosis of any specialist located anywhere. In the context of PACS (Picture Archiving and Communication Systems), the whole medical file of a given patient is instantaneously available. Nevertheless, the image compression techniques often suffer of a lack of essential functionalities: scalability, region of interest coding, security tools.

In classical image encoding systems, the last compression operation consists in entropy coding, generally based on variable length codes. If the coding process produces a single bitstream, as soon as one bit is lost during the transmission of encoded data, the whole image is then lost.

For this reason, progressive transmission of information constitutes a key issue in the domain of telemedicine: several bitstreams representing the complete coded image, and each of them corresponding to one layer of quality and/or resolution of the original image. However, in such a scheme, a bitstream is strongly dependent on the previous decoded ones. Thus lossing one part of the global message prevents from reconstructing the current and next levels of representation. Linked to progressivity, all the bitstreams do not have the same priority level: error-correcting systems have to provide hierarchical protection.

One the other hand, multimedia applications transmit more and more important information volume over packet switch data network as the familiar Internet Protocol. For a wide area network level, any daily Internet weather report shows a huge percentage of packet losses. Hence we propose in this paper an original way of scalable source preservation, by realizing multiple description of information elements in a very low order

Further author information: (Send correspondence to Marie Babel or Benoît Parrein)

M.B.: E-mail: mbabel@insa-rennes.fr, Telephone: +33 (0)2 23238597

B.P.: E-mail: Benoit.Parrein@polytech.univ-nantes.fr, Telephone: +33 (0)2 40683047 


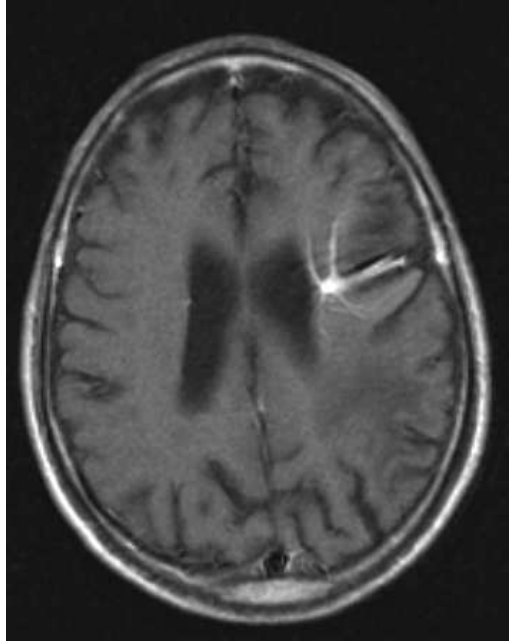

(a) Original image.

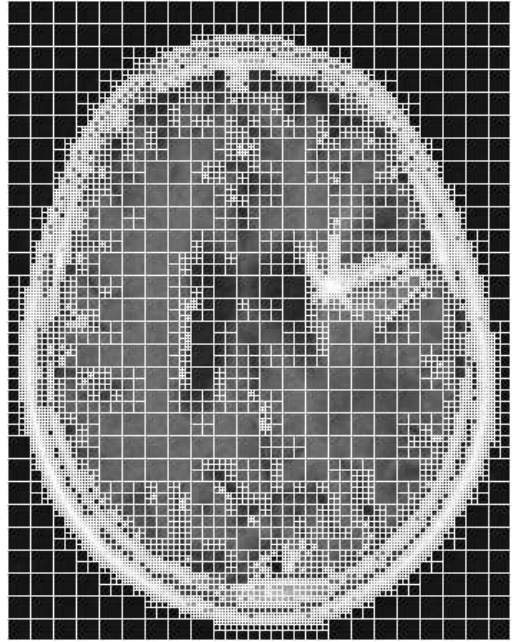

(b) Non-uniform sub-sampling (blocks image)

Figure 1. Visualization of the grid produced by the spatial coder of the LAR method.

of complexity. Thanks to the Mojette transform technique, the descriptions are transmitted without adding any specific mechanism for regulating flows purpose (no packet hierarchy, no integrity packet and no real-time checking).

This paper is organized as follows: the LAR (Locally Adaptive Resolution) image coding method is first presented. The following section attempts to describe the Mojette transform principles. The section 4 explains the joint LAR and Mojette image coding scheme.

\section{LAR METHOD FOR LOSSY/LOSSLESS IMAGE COMPRESSION}

The LAR (Locally Adaptive Resolution) method was first designed for low bit-rate greyscale image coding purpose $^{1}$. Recent researches lead to construct a scalable encoding scheme, based on a pyramidal predictive description, and also efficient for both lossless and lossy compression ${ }^{2}$. Another improvement was realized through self-extracting regions of interest representation. Extensions have also permitted to address middle and high quality encoding and region-level chromatic images encoding. ${ }^{3}$ These three major topics are developed in the following paragraphs.

\subsection{Basic scheme of LAR method}

The LAR method is a two-layer codec: a spatial codec and a complementary spectral one. Indeed, images are then considered to be the superposition of the global information and the texture (local information). The spatial codec is designed to provide a low bit-rate compressed image, whereas the spectral one encodes the texture. Note that the quality of the low resolution LAR image has been evaluated and recognized to be better than JPEG $2000^{4}$.

\subsubsection{Spatial coder}

The basic idea is that the local resolution, i.e. the pixel size, can depend on the local activity. This leads to construct a variable image resolution, determined by means of a quadtree data structure. The final size of pixels (typically for natural images from $16 \times 16$ to $2 \times 2$ ) is computed thanks to a local gradient estimation: each block is reconstructed by its average luminance. Through this decomposition, the pixel size gives implicitly the nature of the blocks. Indeed, small ones are located on contours whereas large ones are situated on smooth areas. These particularities are easily detected on figure 1. 
In a lossy context, this image content information controls a psychovisual quantization of the luminance: large squares require a fine quantization (in uniform areas, human vision is strongly sensitive to brightness variations) and small ones can support a coarse quantization (low sensitivity).

To sum up, this coder has mainly two distinctive characteristics: (i) we have a very fast and efficient technique for high compression ratio, and (ii) the method simplifies the image source by removing the local texture while preserving objects boundaries. Perceptible blocks artifacts in homogenous areas are easily removed by a efficient post-processing: an adaptive interpolation based on theory of optimal recovery is implemented ${ }^{5}$.

\subsubsection{Spectral coder}

In order to obtain higher image quality, the texture (whole error image) can be encoded by the spectral coder that uses a DCT adaptive block-size approach ${ }^{6}$. Both the size and the DC components are here provided by the first coder. The use of adapted square size allows a content-based scalable encoding scheme: for example, edge enhancement can be made by only transmitting the AC coefficients of small blocks.

\subsection{Scalable lossless coding : LAR-APP method}

In medicine, only the losslessly encoded images are recognized legal. In the reference ${ }^{2}$ we have introduced an original minimal redundancy pyramidal decomposition that efficiently exploits our particular quadtree based representation. In this way, two successive scans of the pyramid are applied, so that the blocks image description (see §2.1) is first encoded before processing the texture. This paragraph expounds the principle of the LAR-APP (LAR Pyramidal Predictive Approach) method.

\subsubsection{Spatial coder and general principle of the LAR-APP}

The scalable encoding scheme of the LAR-APP is based on the adaptation of the predictor described by $\mathrm{Wu}^{7}$. For full resolution image, errors are coded by means of three interlaced sampling of the original image. By this way, we tend to obtain a spatial configuration of $360^{\circ}$ type context surrounding a given pixel.

Let the original image be of width $W$ and height $H$. The first stage of our pyramid concerns the image of size $W / 16 \times H / 16$ (level 4). The first pass of the Wu algorithm (simple causal DPCM coding of the block values) is applied. Then, each square is split until its maximal size (given by LAR variable resolution) is reached. For a given level (block size) of the pyramid, the blocks of smaller or equal size are processed by successively the second an third passes of the $\mathrm{Wu}$ predictor. By this way, taking into account the variable resolution LAR image, a progressive content-based method is developed : if a restricted context is sufficient for the large blocks, a more accurate and informative context exists for the small blocks (objects boundaries).

As the level 1 of the pyramid concerns the last level needed to recover the block image (grid and low LAR resolution information), four different bitstreams are required (figure 2).

\subsubsection{Scalable texture encoding}

In order to encode the error image (texture), the previous described scheme is simply extended in order to process the complete pyramid. Our algorithm operates in two steps:

- Half-resolution texture encoding: $16 \times 16,8 \times 8$ and $4 \times 4$ blocks are successively decomposed until the level 1 of our pyramid is reached. Full resolution is obtained thanks to an interpolation process (see figure $3)$.

- Full-resolution error image encoding: Full-resolution texture is then encoded in the previously described manner. Our approach allows to easily distinguish pixels of contours (high activity: higher prediction errors), from flat areas pixels (low activity : lower prediction errors), so that resulting coding cost is drastically decreased. 

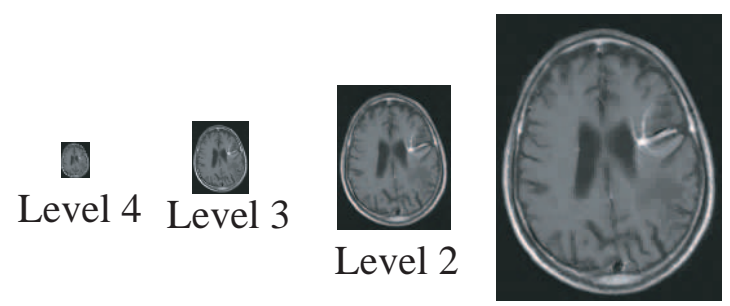

Level 1

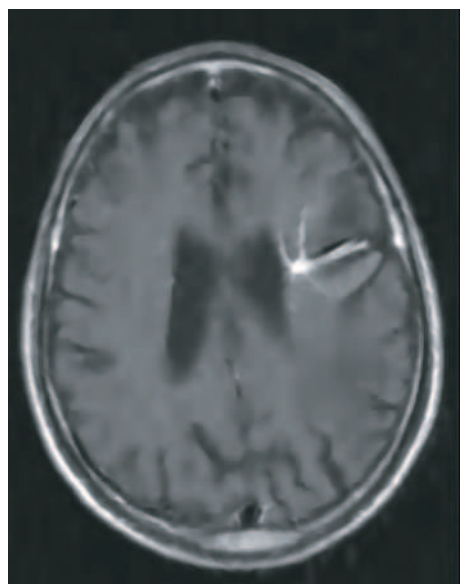

Interpolated image

Figure 2. Original image: $352 \times 448$ ( 8 bpp; 157696 bytes) - Pyramidal decomposition: Level 4 (0.06 bpp; 1182 bytes, including grid information) - Level 3 (0.106 bpp; +906.75 bytes) - Level 2 (0.216 bpp; +2168 bytes) - Level 1 (0.410 bpp; +3824 bytes)
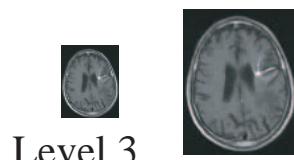

Level 2

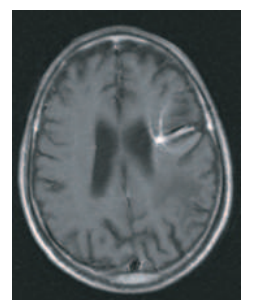

Level 1

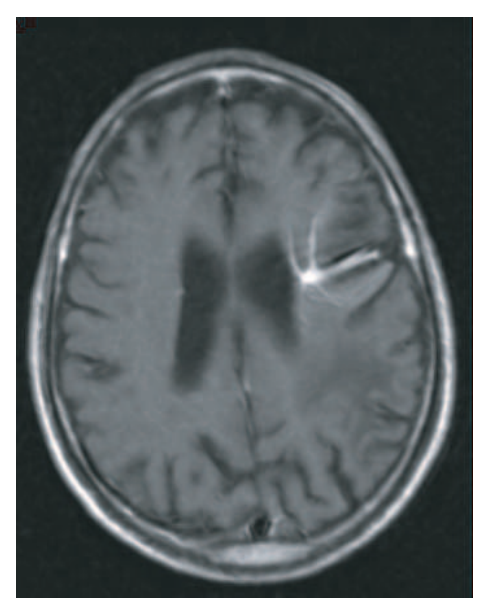

Interpolated image

Figure 3. Second descent process of the pyramid: texture encoding, intermediate images and interpolated one from half-resolution image (1.07 bpp - +13010 bytes). The complete lossless encoding of the image requires 44943 bytes more. 


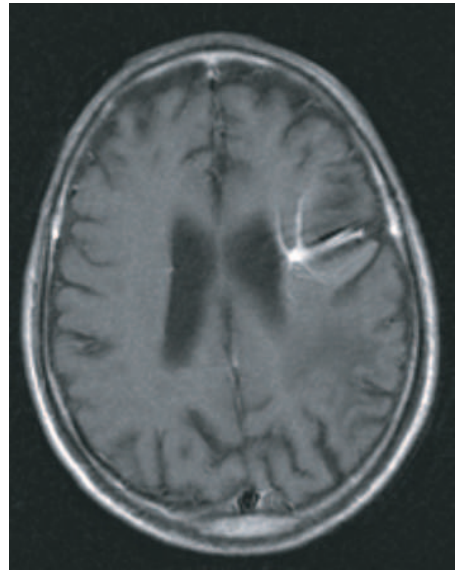

Original image

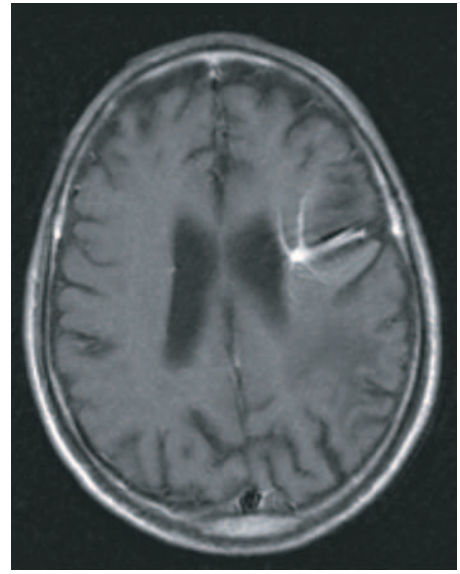

Near-lossless

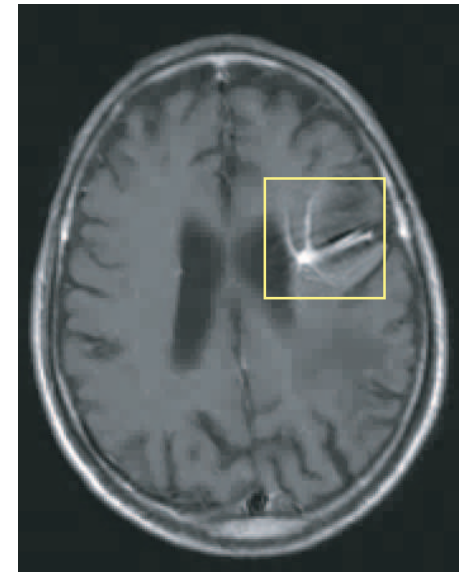

ROI

Figure 4. Near-lossless encoded image (1.966 bpp) and example of the ROI selection result.

\subsubsection{Near-lossless encoding}

The common encoding mode named "near-lossless" consists in applying small quantization to have resulting undetectable errors. Because of the content preservation property of the LAR method, it is easy to adapt quantization to human visual perception. For example, in figure 4, one can find the result of the pyramidal decomposition where a quantization step equal to 4 is applied on contours $(2 \times 2$ blocks), and equal to 2 on flat areas.

\subsection{Region of interest}

Typically, only a small portion in a medical image contains relevant information diagnostically useful, the remaining portion being displayed only as a visual background. Through the definition of a Region of Interest (ROI), images can be globally lossly compressed and locally losslessly encoded. Combined with progressive encoding scheme, this regions scalability allows a faster access to significant data. This subsection introduces the self-extracting region representation technique thanks to a "controlled" segmentation process, so that ROI could be enhanced.

\subsubsection{Self-extracting region representation}

Unlike traditional compression techniques, the low resolution LAR image does not introduce strong distortions which usually prevent from a reliable segmentation process. Indeed, the variable block size representation resulting from a local gradient distance can constitute a "split" step in a segmentation scheme. The original idea of the self-extracting region representation is to transmit the low resolution LAR image, and to continue the segmentation ("merge" step) at both the sender and receiver. As regions are built as union of blocks, two main advantages are introduced by the method:

- a free region representation (no cost overhead for the contours description),

- contours regions and texture coding share the same grid of representation.

\subsubsection{ROI coding}

A ROI can be simply described at both the coder and decoder as a set of regions resulting from the segmentation step. Therefore, only labels of these regions are transmitted. As the ROI is built from the variable block size representation, its enhancement (texture coding) is straightforward because it consists only in executing the LAR-APP codec for the validated blocks, i.e. ROI internal blocks. An example of this scheme is shown in figure 4. 


\section{MOJETTE TRANSFORM}

\subsection{The direct transform}

The Mojette transform ${ }^{8}$ is an exact and discrete Radon transform. It easily describes an image by a finite set of 1 D-projections. Each angle of projection $\theta$ is defined by a couple of integers $(p, q)$, prime each others, with $\frac{q}{p}=\tan \theta$. The direct Mojette transform of an image $f(k, l)$, denoted by $\mathcal{M} f$, represents a set of $N$ projections $\mathcal{M}_{p, q} f$ such as

$$
\mathcal{M} f=\left\{\operatorname{proj}_{p_{i}, q_{i}}, i=1,2 \ldots N\right\}
$$

To compute this transform, we simply use additions in directions determined by the couple of integers $(p, q)$. The resulting projections are formed by a sum of elements set called bins. The value of a projection bin $m$ is calculated by the sum of pixels $f(k, l)$ located on the line determined by $m=-q k+p l$. The following equations stand for the definition of the Mojette-Dirac transform:

$$
\left\{\begin{array}{l}
{\left[\mathcal{M}_{p, q} f\right](m)=\operatorname{proj}(p, q, m)=\operatorname{proj}_{p, q}(m)=\operatorname{proj}_{\theta}(m)} \\
{\left[\mathcal{M}_{p, q} f\right](m)=\sum_{k} \sum_{l} f(k, l) \Delta(m+k q-p l)}
\end{array}\right.
$$

where $\Delta$ is the Krönecker function*.

Since each pixel contributes to one bin, the order of complexity is necessarily of $\mathcal{O}(P Q=I)$ for any projection. If we want to compute a $N$ projections set, the order of complexity is $\mathcal{O}(I N)$, linear in the pixels number and in the projections number.

The Mojette transform is linear with the pixels number and the projections number, as the Fourier and Meyer (wavelet) ones. Contrary to these latter, Radon and Mojette transforms do not aim at obtaining an orthogonal decomposition of the signal, but a frame instead. They produce useful redundancy for reconstruction. In fact, this constitutes the fundamental difference we can find between these two transform families: if the Fourier and Meyer transforms provide a hierarchical coefficients order, the bins resulting from Radon and Mojette transforms application on a signal have all the same weights in the transform domain. However, a given projection carries a quantity of information that is relative to its capacity to recover a portion of the original domain. Moreover, each element contains the mean value (the sum of all projections bins), whereas in case of orthogonal transform, this information is always contained if the first coefficient, but absent in the others.

This fundamental frame property is a very interesting feature for the direct and inverse Mojette transforms implementation: knowing that construction and reconstruction order can be left to the user choice. The frame-like definition allows redundancies that can be further exploited for images description and image communication.

\subsection{The inverse transform}

\subsubsection{Algorithm}

The Mojette transform can be expressed as the product of the transformation matrix $M$ with the vector composed of pixel values $F$. Inverting the Mojette transform is equivalent to resolving the linear system

$$
{ }^{*} \Delta(m)= \begin{cases}1 & \text { if } m=0 \\ 0 & \text { otherwise }\end{cases}
$$


$M \dot{F}=B$ where $B$ is composed of the bin values. However, the transformation matrix $M$ is quite large (number of pixels $\times$ number of bins) and is rectangular and very sparse due to the frame nature of the Mojette.

The Mojette reconstruction algorithm is based on the fact that all bins do not correspond to the same number of projected pixels. It is obvious that the value of a bin corresponding to a single pixel is identical to the pixel value. When such a one-to-one correspondence is found, the pixel value is copied from the bin value. The pixel is then removed from all the projections (i.e. its value is subtracted from all the bins where it projects). At this step, the remaining bins represent the Mojette transform of the unreconstructed part of the image.

Reconstructing the image from a set of projection is then a process that iteratively:

1. finds a reconstructible bin (i.e. a bin projected from a single pixel),

2. "backprojects" its value onto the origin pixel,

3. updates the projections,

until the reconstruction is completed or no one-to-one correspondence between a bin and a pixel is found.

\subsubsection{Reconstruction criterion}

A rectangular image $P \times Q$ can be reconstructed from a set of projections with directions $\left\{\left(p_{i}, q_{i}\right)\right\}$ if and only if:

$$
\left\{\begin{array}{l}
\sum_{i}\left|p_{i}\right| \geq P \\
\text { or } \\
\sum_{i}\left|q_{i}\right| \geq Q
\end{array} .\right.
$$

When building a set of reconstructible projections, we can choose to comply with either of the two conditions. For example, if we set all $q_{i}$ to 1 , a $P \times Q$ image will require exactly $Q$ projections to be reconstructed whatever its width $P$. From this reconstructible set of $Q$ projections, we can build a redundant set by adding new projections. With $N-Q$ extra projections, any subset of $Q$ projections among the total $N$ is enough to reconstruct the image.

\subsection{Multiple description by Mojette transform}

In the framework of communication systems, the Mojette transform can be applied for multiple description (MD) codes. These codes are precisely overviewed in $^{9}$. MD code consists in separating an original source by several independent descriptions which give a degraded but useful version. Making descriptions individually good, yet not too similar, is the fundamental tradeoff of MD coding. The Mojette transform can be simply used to realize this tradeoff ${ }^{10}$. We describe here the mechanism of the packetization strategy that converts the prioritized multiresolution bitstream into an N-packet unprioritized MD packet stream using the Mojette transform.

Each element of a source bitstream is allocated on a 2D support which can be considered as a geometrical buffer in our communication system. The application of the Mojette direct transform on this buffer produces the descriptions. Each description in the MD stream fills an entire network packet, thus the terms descriptions, packets and projections are used indifferently. It can be extended to many input bitstreams. The method which uses several geometrical buffers is described even if these buffers can be concatenated in order to optimize the stretch factor of the code i.e. the transmitted and original information ratio ${ }^{11}$. However, the separated design realizes the protection scheme with a more simple partition of the data. It is based on separable geometrical buffers for each stream and priority. The Mojette transform of each support is then simultaneously computed. The necessary projections number indicates the priority level supported by a buffer. This configuration leads to the same angles of projection and each buffer to construct a transport unit.

Figure 5 illustrates this method. Three rectangular supports have distinct reconstruction properties to obtain three scalable protection levels. Sub-stream 1,2,3 are allocated respectively in geometrical buffer 1, 2 and 3. 


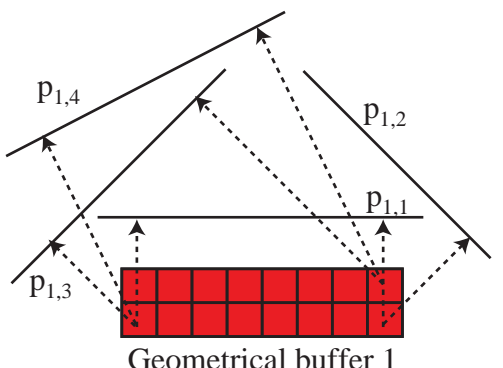

Geometrical buffer 1

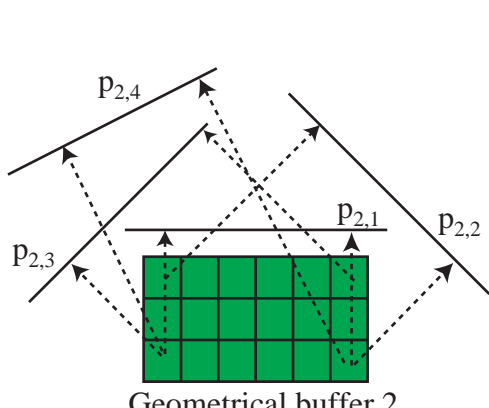

Geometrical buffer 2

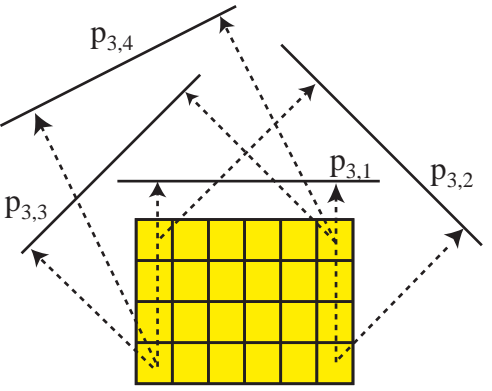

Geometrical buffer 3

Geometrical buffer projections

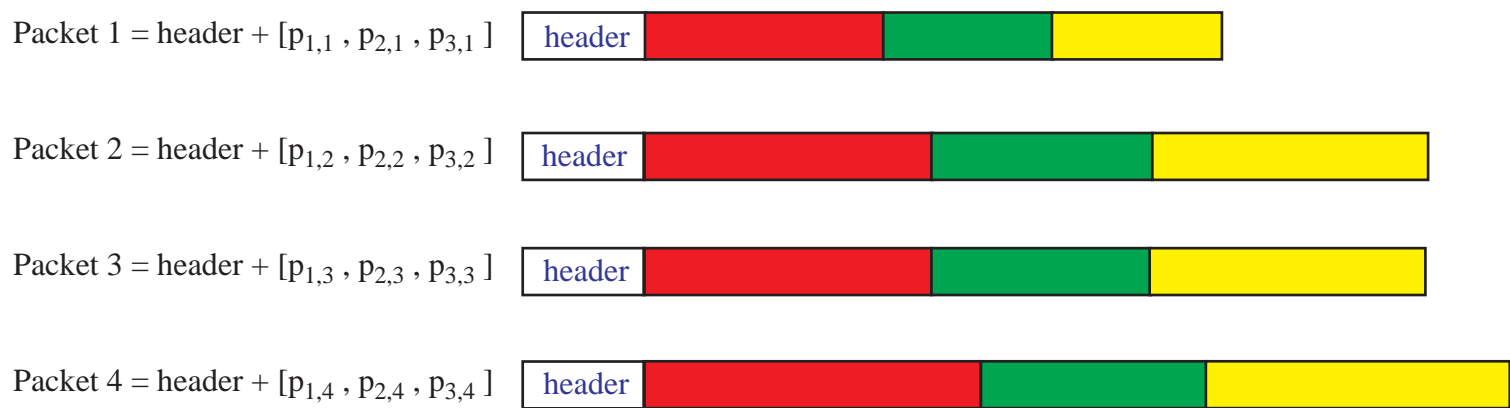

Packets construction

Figure 5. Three levels protection scheme with geometrical buffer separation. One packet construction is realized by concatenating same angle projections. $p_{a, b}$ stands for the $b^{\text {th }}$ projection of the $a^{\text {th }}$ geometrical buffer.

Support 1 (resp. 2 and 3 ) is reconstructible by two projections (resp. three and four) taken in the projection set $S=\{(-2,1),(-1,1),(0,1),(1,1)\}$. The packet 1 contains then all projections of angle $(0,1)$ as packet 4 which contains projections of angle $(-2,1)$. This involves sizes of variable packages while simplifying the insertion of angle labels.

This method presents a solution to protect scalability. The Mojette transform produces descriptions which are equivalent for the transmission network. For one priority level, if the transmission is composed of $M$ projections and $N-M$ redundant projections, it is possible to rebuild in a deterministic way the layer starting from any $M$ packets received among $N$. By this mechanism, we have supported the hierarchy of the source over a best-effort channel, with an end-to-end Quality of Service guarantee.

\section{JOINT LAR AND MOJETTE IMAGE CODING: APPLICATION TO TELEMEDECINE}

Telemedicine applications need simultaneously compression and secured transmission of sensitive data. In some ways, the securization process can also be improved by using progressive method of coding: the supplementary bytes required in order to encode redundancies are larger in case of non-progressive schemes. In this context, this section presents the joint LAR and Mojette technique of image coding.

For encoding a set of $i$ bitstreams with distinct priority levels, we first determine the number of maximal projection, namely $N$ (number of IP packets). The Mojette transform is then executed on each bitstream $i$, 
Table 1. Size of the different table associated to their corresponding number of projection.

\begin{tabular}{|c|c|c|c|c|c|c|}
\hline Bitstream & 1 & 2 & 3 & 4 & 5 & 6 \\
\hline Initial size of bitstreams (bytes) & 1182 & 907 & 2168 & 3824 & 13010 & 44943 \\
\hline Size of layers (bytes) & 394 & 227 & 434 & 638 & 1859 & 5618 \\
\hline Number of necessary projections $\left(H_{i}\right)$ & 3 & 4 & 5 & 6 & 7 & 8 \\
\hline Number of redundant projections $\left(N-H_{i}\right)$ & 5 & 4 & 3 & 2 & 1 & 0 \\
\hline
\end{tabular}

considering the number of necessary projections $H_{i}$, and the number of redundant projections $N-H_{i}$. Final process consists of all projections concatenation as depicted in figure 6 .

The global LAR compressed bitstream is first split into 6 bitstreams, as depicted in table 1 . The first one encodes both the grid and the level 4 of the first multiresolution decomposition (see figure 2). Bitstreams 2, 3 and 4 correspond to respectively level 3,2 and 1 of the same first descent process. Adding the bitstream 5 allows to reconstruct the half-resolution image (level 1 of the second descent - figure 3), and bitstream 6 encodes losslessly the full resolution information. The information collected in table 1, which gives the size of each stream and the associated projections, are illustrated by figure 6: one can easily see that the higher protection level is applied on the first bistream. Indeed, as the grid and the level 4 of the pyramid are essential to decode the image, the securization process proposes to widely overrepresent these information.

In our example, we have defined $N=8$. The 1182 bytes of bitstream 1 are represented by a $3 \times 394$ rectangle. Note that this priority encoding system allows the reconstruction of the level 4 image as soon as $H_{1}=3$ IP packets among the $N=8$ transmitted ones are received at the decoder. If one considers the symbols of information level rather than the packet level, the Mojette transform is very close to the optimal reconstruction: indeed, it only requires $2 \%$ of additional symbols in comparison with the initial volume to carry out for complete decoding $^{\dagger}$. The present redundancy corresponds to an overhead of 7326 bytes, i.e. $11 \%$ of global compressed data, distributed among 8 IP packets. The unequal error protection strategy have to be compared to the equal one. In the latter case, the extra cost would correspond to an unique redundant projection, so that the initial volume (losslessly encoded image) is represented by a $9 \times 7326$ rectangle.

Figure 7 shows the quality level of reconstructed images according to reception rate. For an equal protection level, the reception of $90 \%$ of data (9 IP packets among the 10 transmitted ones) ensures the complete recovering of original image. However the lost of more than one packet via the Internet prevents from visualizing any image at the decoder. This is of course unacceptable within the framework of emergency medicine. This is the reason why scalable technique with unequal error protection such as the one presented above suits better to telemedicine processes.

\footnotetext{
${ }^{\dagger}$ If the initial volume consists of $k$ symbols, the inversion of the Mojette tranform requires only $(1+\epsilon) k$ symbols where $\epsilon=0,02$.
}

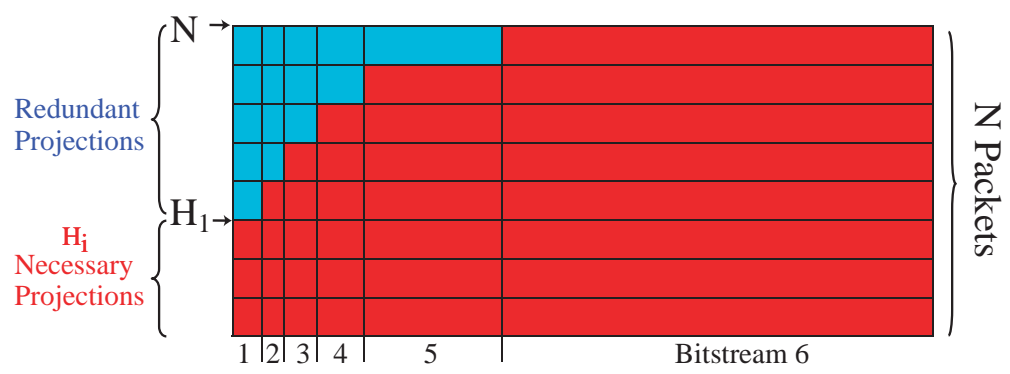

Figure 6. IP packets representation : visualization of packet size, number of necessary and redundant projections per bitstream. 


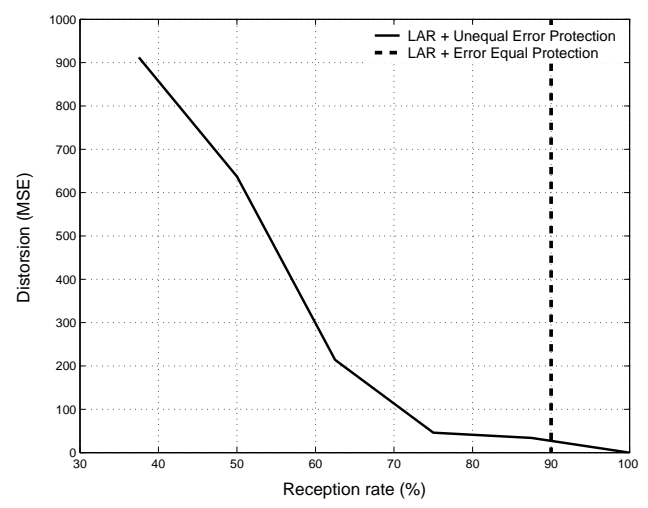

Figure 7. Reconstructed image quality for different reception rates. $100 \%$ means that all projections are received.

\section{DISCUSSION}

The progressive transmission of multimedia contents has emerged from the mandatory use of old narrow-bands as ISDN or GSM to transmit huge real-time data. It can be expected in the near future a better accordance between data volume and its transmission. First, very high bit rates are promised by new network technologies as UMTS for mobile transmission or are already here like fibre length wave multiplexing. Second, as demonstrated here with the LAR codec, there are still great achievements to be obtained in terms of multimedia compression as soon as proper models of the scene can be introduced. These facts lead to think that progressive transmission will not be so useful when the source and the channel will have meet common objectives. However, it can be argued that progressive transmission will still stands in the future. A basic explanation for that is the ad hoc hierarchy derived from the client's needs as the keypoint in the design of the service. When one first gets the sorted information for a given task and also at the same time optimizes the usage from the final user, this information can be considered as metadata shaping exchanges. This is obviously true for the transmission but as well for storage or services. For instance, a hierarchical multimedia storage will still be of great use (even if mass storage online becomes very cheap) because specific requests associating with the service will be performed onto the first levels of hierarchy. This is specifically true for telemedicine when the future collection of data for a patient (respectively for a disease) will become enormous. The request for a specific disease suspicion of the patient should not lead to extract the whole patient case but only relevant ones (an X-ray of the leg is not relevant for a lung cancer).

These hierarchy labels used as metadata allow for speeding up requests and information extraction. In this respect, the introduction of the metadata inside the data (using a watermarking technique for instance) can value the hierarchy of a given multimedia service or coder. In summary, it should be stressed that the possible extracted hierarchy must be rich enough to respond to a wide variety of medical tasks. This is the case with the LAR coder.

The agreement between the LAR coder and the telemedicine field being appropriated, the channel constraints are to be stressed via the use of the Mojette transform. The center of the discussion is then to use either an unequal error protection scheme (UEP) or an equal error protection scheme (EEP). In a packet transport mode, the use of forward error correcting (FEC) codes like Reed-Solomon (RS) codes authorizes the loss of units without compromising the rendering of the information. Their capacity of correction for the whole stream can in certain case reach the following property: it is possible to rebuild the original stream in a deterministic way as the $M$ packets messages starting from any $M$ packets received among $N$. That describes the EEP: one equal level of redundancy for the whole stream. Decoding fails if more than $N-M$ packets are lost. When the exact rebuilding is impossible, it can be advantageous to want to compensate for the errors rather than to correct them. It is all the interest of UEP which can deliver a degraded version when the transmission is particularly disturbed. For instance, FEC codes are implemented with different stretch factors to deliver a JPEG2000 stream on a wireless link. ${ }^{12}$ By employing this scheme and not the EEP, the source can consider a great diversity of recipients with only one coding. UEP by FEC codes is therefore one multiple description implementation. 
In the unequal protection framework, the contribution of the Mojette transform with respect to the traditional FEC as RS is for the adjustments of priority levels (of redundancy). The transform offers a greater flexibility knowing than RS codes are strongly constrained by the algebra of the Galois fields. So the Mojette transform supports better way the highly granular hierarchy which is delivered by LAR coding.

\section{CONCLUSION}

The LAR image coding method is a new scalable method: not only efficient in terms of image quality for low bit-rates, it also achieves excellent compression ratio for losslessly encoded images. Each bitstream is associated to both a particular resolution level of the pyramid and a specific type of information (smooth areas or contours). Knowing that, loosing a given bitstream leads to reconstruct the image at a limited resolution level. The LAR coder has the advantage of recovering low bit-rates encoded images with good visual quality (e.g. no blocks or ringing effect).

The Mojette transform is then applied on each compressed bitstream provided by the LAR coder: a hierarchical cost-controlled protection is so available. All the projections and added redundancies are dispatched among the whole of IP packets. Thus, as the Mojette cost does only depend on the number of received packets and not on their contents, the decoder immediately launches a reconstruction process upon reception of each packet.

An original joint source-channel coding, based on LAR compression and Mojette transform, has been especially designed for Internet transmissions. In the application presented in section 4, a LAR encoder feeds data to the Mojette transformer which behaves as a priority encoding system. The association of the LAR with the priority encoding system by Mojette transform provide a end-to-end Quality of Service guarantee.

\section{REFERENCES}

1. O. Deforges and J. Ronsin, "Nonuniform Sub-Sampling using Squares Elements : a Fast Still Image Coding at Low Bit-Rate," International PCS'99 , 1999.

2. M. Babel, O. Déforges, and J. Ronsin, "Lossless and Lossy Minimal Redundancy Pyramidal Decomposition for Scalable Image Compression Technique," in 4th IEEE International Conference on Multimedia and Expos , ICME'03, 3, pp. 161-164, (Baltimore, USA), July 6-9 2003.

3. O. Deforges and J. Ronsin, "Supervised Segmentation at Low Bit Rates for Region Representation and Color Image Compression," Proc. ICME'2002, 2002.

4. O. Deforges and J. Ronsin, "Region of Interest Coding for Low Bit-Rate Image Transmission," Proc. ICME'2000 , 2000.

5. D. D. Muresan and T. W. Parks, "Optimal Recovery Approach to Image Interpolation," Proc. IEEE ICIP, Greece, p. Image Interpolation and Spatial Filtering, 2001.

6. C. Chen, "Adaptive transform coding via quad-tree based variable block-size DCT," in ICASSP'89, pp. 1854-1856, 1989.

7. X. Wu, "Lossless Compression of Continuous-Tone Images, via Context Selection, Quantization and Modelling," IEEE Trans. on Image Processing 6, pp. 656-664, May 1997.

8. J. Guédon, A Discrete Tomograph: the Mojette Transform (the Shape and the Projection). HDR (in french), University of Nantes, 2001.

9. V. Goyal, "Muliple Description Coding : Compression Meets the Network," IEEE Signal Processing Magazine 18, pp. 74-93, September 2001.

10. B. Parrein, N. Normand, and J. Guédon, "Multimedia Forward Error Correcting Codes," Annals of Telecommunications 58, pp. 448-463, April 2003.

11. B. Parrein, Multiple Description of Information by Mojette Transform. PhD thesis (in french), University of Nantes, November 2001.

12. JPEG. Editors, "ISO/IEC CD 15444-11 / ITU-T SG8 JPEG 2000 image coding system - Part 11: Wireless JPEG2000 Committee Draft," 2004. 